\title{
Precision Penning trap mass measurements on exotic ions: status and perspectives
}

\author{
Klaus Blaum • Michael Block
}

Published online: 26 August 2009

(C) The Author(s) 2009. This article is published with open access at Springerlink.com

\begin{abstract}
Penning traps are powerful instruments for the precise and accurate mass determination of rare isotopes. At present, many Penning trap facilities installed at radioactive beam facilities provide key data for nuclear astrophysics, for the study of nuclear structure evolution far from stability, and the test of fundamental interactions. This article summarizes the present status and current limits in the field of high-precision Penning trap mass measurements on short-lived exotic ions.
\end{abstract}

Keywords Penning trap • Mass spectrometry $\cdot$ Exotic ions

\section{Introduction}

Nowadays relative mass precisions of $\delta m / m=10^{-8}$ can be routinely achieved on short-lived nuclides with half-lives in the range of $T_{1 / 2}=100 \mathrm{~ms}$ and production yields of about 100 ions per second employing Penning trap mass spectrometry. Of course the resolving power and thus the accuracy relies on a sufficiently long measurement and storage time. The key ingredient to that are proper cooling, manipulation and detection techniques, which are described in detail in [1]. Therefore, we concentrate in the following on the basics of Penning trap mass spectrometry, its applications and limitations.

K. Blaum $(\bowtie)$

Max-Planck-Institute for Nuclear Physics, Saupfercheckweg 1, 69117 Heidelberg, Germany e-mail: klaus.blaum@mpi-hd.mpg.de

M. Block

GSI Helmholtzzentrum für Schwerionenforschung GmbH,

Planckstrasse 1, 64291 Darmstadt, Germany

e-mail:m.block@gsi.de 


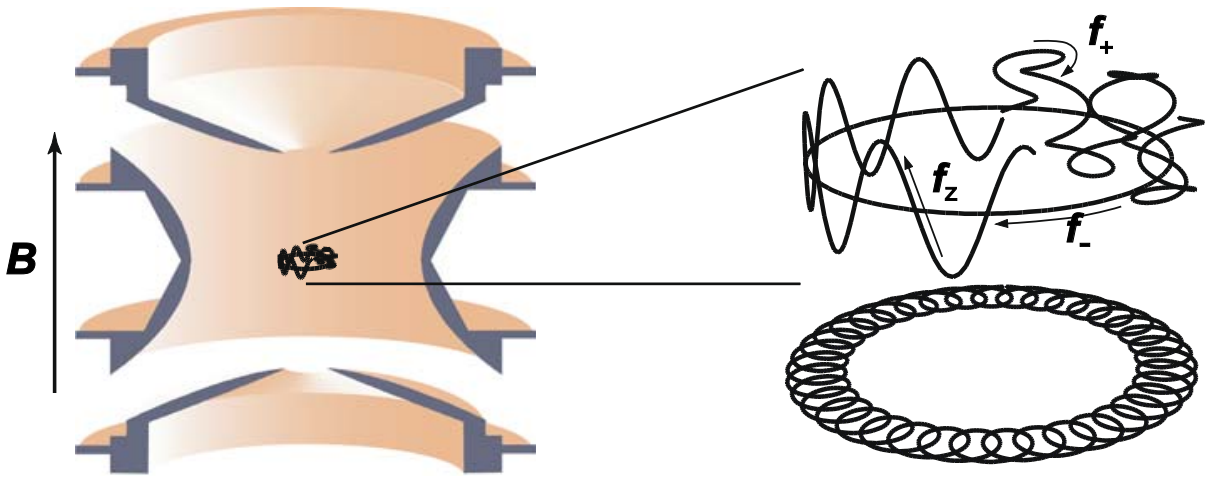

Fig. 1 Left: Sketch of a Penning trap with a diameter of about $2 \mathrm{~cm}$. Right: Trajectory of a charged particle inside the trap with a typical motional amplitude of $1 \mathrm{~mm}$

\section{Mass measurement technique with Penning traps}

In a Penning trap a superposition of a strong homogeneous magnetic field $B$ with a weak electrostatic quadrupolar field $E$ is used to confine the ions three-dimensionally in space (see Fig. 1). The orientation is chosen in a way such that the magnetic field takes care on the radial confinement and the electrostatic field on the axial one, respectively. The ions with charge-to-mass ratio $q / m$ perform a radial circular motion with the mass-characteristic cyclotron frequency of revolution

$$
f_{c}=\frac{1}{2 \pi} \frac{q B}{m} .
$$

In addition, this combination of fields leads to a low-frequency circular drift motion centered in the trap axis, as shown in Fig. 1. Thus, the ion motion is a superposition of three independent harmonic modes with the corresponding magnetron $\left(f_{-}\right)$, modified cyclotron $\left(f_{+}\right)$and axial frequency $\left(f_{z}\right)$ [2]. For an ideal Penning trap the simple relation

$$
f_{c}=f_{+}+f_{-}
$$

holds. The free cyclotron frequency $f_{c}$ of short-lived nuclides is determined at all on-line Penning trap mass spectrometers by using the so-called time-of-flight ion cyclotron resonance (TOF-ICR) detection technique [3]. To this end the ion gets excited with a frequency $f_{R F}$ close to $f_{c}$ and subsequently ejected into a drift region towards an ion detector. The frequency is scanned and finally the mass of the ion of interest determined from the center of the time-of-flight resonance at $f_{c}$. An important prerequisite for an accurate cyclotron frequency measurement is the preparation of cooled and purified ion samples. This is accomplished utilizing radiofrequency ion beam coolers [4] in combination with preparation Penning traps where a mass-selective buffer gas cooling technique [5] is applied to separate nuclear isobars. 


\section{Field of application}

There is a large variety of applications for precision mass values of radionuclides with required relative uncertainties of the measured mass $\delta m / m$ ranging from $10^{-5}$ in chemistry down to below $10^{-8}$ in weak interaction studies and Standard Model tests. Some of these applications will be addressed in the following:

Nuclear structure: Mass measurements in long isotopic and isotonic chains allow the study of the fine structure of the mass surface and to clarify discontinuities in order to extract nuclear structure information from binding energies, as recently performed around the doubly-magic ${ }^{208} \mathrm{~Pb}$ [6]. Mass measurements also play a crucial role for the identification of halo nuclei such as ${ }^{11} \mathrm{Li}$ whose mass has been recently determined with the TITAN system [7]. The superb resolving power of Penning trap mass spectrometers of $10^{6}$ and above allows in addition the resolution [8] and even discovery of low-lying isomeric states [9].

Mass formula: Since no exact description of the strong interaction exists and due to the complexity of the many-body nucleonic system, nuclear masses or more precise nuclear binding energies cannot be predicted by ab initio theories. However, there are a number of phenomenological (macroscopic-microscopic) mass models or mass formulas on the market, which allow a quantitative prediction of atomic masses [10]. They make use of a set of free parameters, which have to be constrained by fitting to experimental data. To this end precision Penning trap mass measurements far from the valley of $\beta$-stability allow for tests of the predictive power of the models and formulas, as discussed in [10] and recently tested e.g. for the $N=82$ shell gap in [11].

Astrophysics: The properties of atomic nuclei, especially their masses, play a crucial role for fundamental questions at the interface of nuclear and astrophysics. Among them the question about the explanation of the elemental and isotopic abundance distribution in the solar system. Also for the understanding of the rprocess, one of the greatest challenges of modern nuclear astrophysics [12], masses are of utmost importance. Very recently a large number of high-precision mass values in the vicinity of the rp-process and the $v$ p-process paths have been obtained at the JYFLTRAP (Jyväskylä, Finland) and SHIPTRAP (GSI Darmstadt, Germany) facilities in order to understand these nucleosynthesis processes better [13]. Mass measurements related to nuclear astrophysics have also been performed with LEBIT (East Lansing, USA) [14] and CPT (Argonne, USA) [15].

Fundamental studies: Direct Penning trap mass measurements with relative uncertainties of $10^{-8}$ and below on superallowed $\beta$-emitters and their daughters contribute to tests of two fundamental postulates of the Standard Model, namely of the conserved-vector-current hypothesis of the weak interaction and the unitarity of the Cabibbo-Kobayashi-Maskawa quark-mixing matrix [16]. Recent mass measurements in this context have been performed, for example, on ${ }^{26} \mathrm{Al}^{m}[17],{ }^{38} \mathrm{Ca}[18,19],{ }^{42} \mathrm{Sc}$ [17], ${ }^{46} \mathrm{~V}[17,20],{ }^{50} \mathrm{Mn}$, and ${ }^{54} \mathrm{Co}[21]$. 


\section{Present status}

Mass range: Penning trap mass spectrometry can be applied to any ion independent of its mass. However, for heavier ions the lower cyclotron frequency results in a lower resolving power at constant excitation time $T_{R F}$ in comparison to light ions. For very light ions the beam preparation that typically requires cooling in an RFQ beam cooler, for example, becomes less efficient even if hydrogen is used as the buffer gas instead of helium. Nonetheless, the mass of the short-lived radionuclide ${ }^{8} \mathrm{He}$ has been recently measured with the TITAN Penning trap mass spectrometer [22]. The heaviest radionuclide ever studied in a Penning trap is ${ }^{254}$ No that was recently investigated at SHIPTRAP.

Resolving power: The mass resolving power $R$ achieved with the TOF-ICR method is given by the cyclotron frequency and the excitation time $T_{R F}$ according to $R=$ $f_{c} T_{R F}$. For a system with $B=7 T$ a resolving power of $R \approx 10^{6}$ can be reached for $A=100$ with an excitation time of $T_{R F}=1 \mathrm{~s}$. For rare isotopes the achievable resolving power is limited by their half-life $\left(T_{R F} \leq 3 T_{1 / 2}\right)$. The resolving power can be further increased using a higher magnetic field or higher charge states. Up to now highly charged ions have only been used for mass measurements of stable ions [23]. Alternative approaches to increase the resolving power are the use of an octupolar excitation [24] and the application of a Ramsey excitation scheme [25].

Accuracy: The statistical uncertainty $\delta m / m$ depends on the resolving power and the number of detected ions $N$

$$
\frac{\delta m}{m} \propto \frac{1}{R \sqrt{N}} .
$$

Typically at least 100 ions are needed to observe a resonance. For $R=10^{6}$ and $N=100$ a relative statistical uncertainty of $10^{-7}$ can be achieved from a single resonance. For rare isotopes the low production rate limits the number of ions that can be obtained in a typical measurement time of few hours per resonance. Thus, a high resolving power is important for a high precision. The final uncertainty of a mass measurement includes systematic effects that may lead to frequency shifts. These have been studied in detail [2,3] and comprise, for example, inhomogeneities of the confining fields, misalignments, and ion-ion interaction. The uncertainty due to temporal changes of the magnetic field limits the measurement time between calibrations to a few hours. The impressive progress in the accuracy of Penning trap mass spectrometry of rare isotopes is illustrated by the mass of the short-lived nuclide ${ }^{38} \mathrm{Ca}\left(T_{1 / 2}=440 \mathrm{~ms}\right)$ which is presently known with a relative uncertainty of $\delta m / m=8 \times 10^{-9}[18]$.

Half-life: Mass measurements of short-lived nuclides are constrained by the time needed for beam preparation. The time for beam cooling, bunching, and isobar separation sums up to some $10 \mathrm{~ms}$. This results in an accessible half-life range of about $T_{1 / 2} \approx 50 \mathrm{~ms}$. The exact number depends on the available yield for a given nuclide and the decay losses that can be tolerated during the preparation and measurement time. The limited resolving power as discussed above favors measurements on light ions. Recently, the mass of the halo nucleus ${ }^{11} \mathrm{Li}$ with a half-life of only $T_{1 / 2}=8.8 \mathrm{~ms}$ 
has been determined with the TITAN system [7] making it the shortest-lived nuclide studied in a Penning trap.

Efficiency: Operating in ultrahigh vacuum and with the application of cooling ion traps allow the precise manipulation of single ions on the quantum level and have an intrinsically high efficiency. The overall efficiency of a Penning trap mass spectrometer for rare isotopes is thus limited by the preparatory steps needed to reduce the energy of the rare isotopes produced in nuclear reactions to a few eV and provide them as cooled, bunched and purified beams. The overall efficiency of typical Penning trap systems is on the percent level. The rare isotope with the lowest production rate for that a mass measurement has been performed with a Penning trap is ${ }^{252}$ No delivered at a rate of only 0.6 particles per second in a recent experiment at SHIPTRAP.

\section{Future perspectives}

New developments will further extend the reach of Penning trap mass spectrometry. The introduction of non-destructive detection techniques with single-ion sensitivity will give access to rare isotopes with very low production rate. The use of Electron Beam Ion Traps optimized for fast and efficient charge breeding will allow a higher accuracy and help to access very short-lived nuclides. Moreover, the completion of next generation radioactive beam facilities will provide even more exotic nuclei with yields sufficient for high-precision mass spectrometry. Thus, more accurate mass values of exotic nuclei even further away from stability will become available in the near future. They will give new insight in the nuclear structure evolution of very neutron-rich nuclei and improve our understanding of the astrophysical r-process.

Acknowledgements Financial support by the Max Planck Society, by the HGF under contract VH-NG-037 and by the German BMBF under contract 06MZ215 is gratefully acknowledged.

Open Access This article is distributed under the terms of the Creative Commons Attribution Noncommercial License which permits any noncommercial use, distribution, and reproduction in any medium, provided the original author(s) and source are credited.

\section{References}

1. Blaum, K.: High-accuracy mass spectrometry with stored ions. Phys. Rep. 425, 1-78 (2006)

2. Brown, L.S., Gabrielse, G.; Geonium theory: physics of a single electron or ion in a Penning trap. Rev. Mod. Phys. 58, 233-311 (1986)

3. Bollen, G., Moore, R.B., Savard, G., Stolzenberg, H.: The accuracy of heavy-ion mass measurements using time of flight-ion cyclotron resonance in a Penning trap. J. Appl. Phys. 68, 4355-4374 (1990)

4. Herfurth, F., et al:: A linear radiofrequency ion trap for accumulation, bunching, and emittance improvement of radioactive ion beams. Nucl. Instrum. Methods A 469, 254-275 (2001)

5. Savard, G., et al.: A new cooling technique for heavy-ions in a Penning trap. Phys. Lett., A 158, 247-252 (1991)

6. Weber, C., et al.: Atomic mass measurements of short-lived nuclides around the doubly-magic ${ }^{208}$ Pb. Nucl. Phys., A 803,1-29 (2008)

7. Smith, M., et al.: First Penning-trap mass measurement in the millisecond half-life range: the halo nucleus ${ }^{11}$ Li. Phys. Rev. Lett. 101, 202501 (2008) 
8. Van Roosbroeck, J., et al.: Unambiguous identification of three $\beta$-decaying isomers in ${ }^{70} \mathrm{Cu}$. Phys. Rev. Lett. 92, 112501 (2004)

9. Block, M., et al.: Discovery of a nuclear isomer in ${ }^{65} \mathrm{Fe}$ with Penning trap mass spectrometry. Phys. Rev. Lett. 100, 132501 (2008)

10. Lunney, D., Pearson, J.M., Thibault, C.: Recent trends in the determination of nuclear masses. Rev. Mod. Phys. 75, 1021-1082 (2003)

11. Dworschak, M., et al.: Restoration of the $N=82$ shell gap from direct mass measurements of ${ }^{132,134}$ Sn. Phys. Rev. Lett. 100, 072501 (2008)

12. Pfeiffer, B., Kratz, K.-L., Thielemann, F.-K., Walters, W.B.: Nuclear structure studies for the astrophysical r-process. Nucl. Phys., A 693, 282-324 (2001)

13. Weber, C., et al.: Mass measurements in the vicinity of the rp-process and the $v$ p-process paths with JYFLTRAP and SHIPTRAP. Phys. Rev., C 78, 054310 (2008)

14. Schury, P., et al.: Precision mass measurements of rare isotopes near $N=Z=33$ produced by fast beam fragmentation. Phys. Rev., C 75, 055801 (2007)

15. Fallis, J., et al.: Determination of the proton separation energy of Rh-93 from mass measurements. Phys. Rev., C 78, 022801 (2008)

16. Towner, I.S., Hardy, J.C.: Improved calculations of the isospin-symmetry-breaking corrections to superallowed Fermi $\beta$ decay. Phys. Rev., C 77, 025501 (2008)

17. Eronen, T., et al.: $Q$ values of the superallowed beta emitters ${ }^{26} \mathrm{Al}^{m},{ }^{42} \mathrm{Sc}$, and ${ }^{46} \mathrm{~V}$ and their impact on $V_{u d}$ and the unitarity of the Cabibbo-Kobayashi-Maskawa matrix. Phys. Rev. Lett. 97, $232501(2006)$

18. Bollen, G., et al.: Experiments with thermalized rare isotope beams from projectile fragmentation: a precision mass measurement of the superallowed beta emitter Ca-38. Phys. Rev. Lett. 96, 152501 (2006)

19. George, S., et al.: Ramsey method of separated oscillatory fields for high-precision Penning trap mass spectrometry. Phys. Rev. Lett. 98, 162501 (2007)

20. Savard, G., et al.: Q value of the superallowed decay of V-46 and its influence on V-ud and the unitarity of the Cabibbo-Kobayashi-Maskawa matrix. Phys. Rev. Lett. 95, 102501 (2005)

21. Eronen, T., et al.: $Q_{E C}$ values of the superallowed beta emitters ${ }^{50} \mathrm{Mn}$ and ${ }^{54} \mathrm{Co}$. Phys. Rev. Lett. 100, 132501 (2008)

22. Ryjkov, V.L., et al.: Direct mass measurement of the four-neutron halo nuclide ${ }^{8} \mathrm{He}$. Phys. Rev. Lett. 101, 012501 (2008)

23. Schuch, R., et al.: Precise atomic masses for fundamental physics determined at SMILETRAP. Adv. Quantum Chem. 53, 67-81 (2008)

24. Eliseev, S., et al.: Octupolar excitation of ions stored in a Penning trap mass spectrometer-a study performed at SHIPTRAP. Int. J. Mass Spectrom. 262, 45-50 (2007)

25. George, S., et al.: Separated oscillatory fields for high-precision Penning trap mass spectrometry. Int. J. Mass Spectrom. 264, 110-121 (2007) 